\title{
High Speed Dim Air Target Detection Using Airborne Radar under Clutter and Jamming Effects
}

\author{
Amir ALMSLMANY $Y^{1}$, Qunsheng $C A O^{1}$, Caiyun $W A N G^{2}$ \\ ${ }^{1}$ College of Electronic and Information Engineering, Nanjing University of Aeronautics \& Astronautics, 29 Yudao St., \\ Nanjing 210016, China \\ ${ }^{2}$ College of Astronautics, Nanjing University of Aeronautics \& Astronautics, 29 Yudao St., Nanjing 210016, China
}

a.mslmany@nuaa.edu.cn, qunsheng@nuaa.edu.cn,wangcaiyun@nuaa.edu.cn

\begin{abstract}
The challenging potential problems associated with using airborne radar in detection of High Speed Maneuvering Dim Target (HSMDT) are high noise, jamming and clutter effects. The problem is not only how to remove clutter and jamming but as well as the range migration and Doppler ambiguity estimation problems due to high relative speed between the targets and airborne radar. Some of recently published works ignored the range migration problems, while the others ignored the Doppler ambiguity estimation. In this paper a new hybrid technique using Optimum Space Time Adaptive Processing (OSTAP), Second Order Keystone Transform (SOKT), and the Improved Fractional Radon Transform (IFrRT) was proposed. The OSTAP was applied as anti-jamming and clutter rejection method, the SOKT corrects the range curvature and part of the range walk, then the IFrRT estimates the target' radial acceleration and corrects the residual range walk. The simulation demonstrates the validity and effectiveness of the proposed technique, and its advantages over the previous researches by comparing its probability of detection with the traditional methods. The new approach increases the probability of detection, and also overcomes the limitation of Doppler frequency ambiguity.
\end{abstract}

\section{Keywords}

Airborne radar, dim target detection, space time adaptive processing, jamming

\section{Introduction}

High speed air moving targets detection capability of the airborne radar system has been a critical technique in the community of radar. Brennan and Reed introduced space-time adaptive processing (STAP) to the airborne radar in 1973 [1], the STAP used to reject clutter, and became a crucial technique for improving detection performance of a desired target in the midst of noise, the clutter and the manmade jamming. A vast portion of the research effort has been devoted to the airborne STAP. Most of them are concerned about how to reduce the number of the secondary data, how to enhance the capability of detecting the slowly moving target, the computational complexity and the sample limitation for estimating the clutter covariance matrix, and so on; such as reduced-dimension (RDSTAP) [2], [3], reduced-rank (RR) [4], time varying spacetime auto regressive filtering (TV-STAR) [5], and the extend factor approach (EFA) [6].

However, the problems of range migration compensation, and the Doppler ambiguity estimation are discussed less. Range migration means that the echoes of the moving targets are distributed in adjacent range resolution cells in one coherent processing interval (CPI) which occurs due to the high relative velocity between the radar and the moving target. Such situation may be the case of detecting the fastmoving target in the broadside area, or the moving target in the squint area. For high range resolution (HRR) radar, the range migration, and Doppler ambiguity estimation of the high speed target are more serious. Because the STAP method usually only employs the data in the same range bin, the detection performance for the moving target with range migration is deceased. To improve the detection performance in this case, the range migration, and Doppler ambiguity estimation should be compensated efficiently [7]. Detection methods for space moving targets include the incoherent integration and the coherent integration [8]. For the incoherent integration methods such as radon Fourier transform (RFT) [9], [10], and Hough Transform (HT). However, these methods can only obtain a coarse estimation of the target trajectory without phase information and have a heavy computational burden. On the other hand, the coherent integration methods such as time frequency analysis, keystone transform (KT) [11-14], and likelihood ratio test (LRT), have the optimal detection performance since they make the best use of a large amount of pulse information.

Zhou et al. in [15] combined the STAP/KT in order to detect the ground moving targets, but they ignored the Doppler ambiguity estimation problem, also this method is only valid for ground moving targets where the speed is limited and the clutter effect is lower than air targets. Jia Qiong et al. [16-18] developed a combination technique of KT and the STAP to detect fast air moving dim targets, and 
furthermore compensate the target's range migration by using a filter bank, but also ignoring the Doppler ambiguity estimation problems. Tian et al. [19] proposed a combination of the second-order Keystone transform (SOKT) and the fractional Fourier transform (FrFT), and (RFT) for range migration and Doppler ambiguity compensation but they ignored the jamming and clutter effects. Recently G. Sun et al. [20] proposed a method that uses SOKT to eliminate the quadratic range cell migration (QRCM) and the modified fractional Radon transform (MFrRT) to estimate the ambiguity number. They used the MFrRT to improve the ambiguity estimation, but this method is limited for only low signal to noise ratio (SNR) and ground moving targets in synthetic aperture radar (SAR) applications, with limited speed to $150 \mathrm{~m} / \mathrm{s}$. They ignored the jamming effect, and they didn't discuss how they removed the clutter (note that the clutter effect in case of detecting ground targets is lower than its effect in case of detecting air targets).

In this paper, a new hybrid technique using Optimum Space Time Adaptive Processing (OSTAP), SOKT, and the Improved Fractional Radon Transform (IFrRT) has been proposed in order to tackle all problems, and improving the detection performance for the High Speed Maneuvering Dim Target (HSMDT). First, we apply the OSTAP for the clutter suppression and the anti-jamming, subsequently employ the SOKT to correct the range migration or range curvature, and afterwards utilize the IFrRT to estimate the targets' radial acceleration, and correct the Doppler ambiguity and residual range walk.

The new contributions from this work are, first, applying OSTAP to increase the SNR, to overcome the limitation of [20] that is only applicable in lower SNR levels, second, taking into consideration all the interference effects (jamming, clutter, noise) not only clutter in [20], third, using the combination of SOKT/IFrRT for range migration, and Doppler ambiguity estimation compensation with lower complexity than using SOKT/FrFT/RFT in [19], fourth, the first time to apply these methods in signal processing (monostatic radar) not only in image processing (SAR) [19], [20]. The simulation results validate the proposed method by comparing the probability of detection of (OSTAP /SOKT/IFrRT) with the recently references, (SOKT /MFrRT) [20], (SOKT/FrFT/RFT) [19], (RFT) [10], (STAP) [21], and without applying any method.

The problem formulation and modeling are discussed in Sec. 2. The complete analysis of the OSTAP for antijamming and clutter suppression was demonstrated in Sec. 3. The parameters estimation' algorithm was clarified in Sec. 4. The simulation and result was introduced in Sec. 5, and finally the work was concluded in Sec. 6.

\section{Problem Formulation and Modeling}

As illustrated in the introduction, the most significant problems associated with using airborne radar in detecting the HSMDT can be divided into two main parts, the first

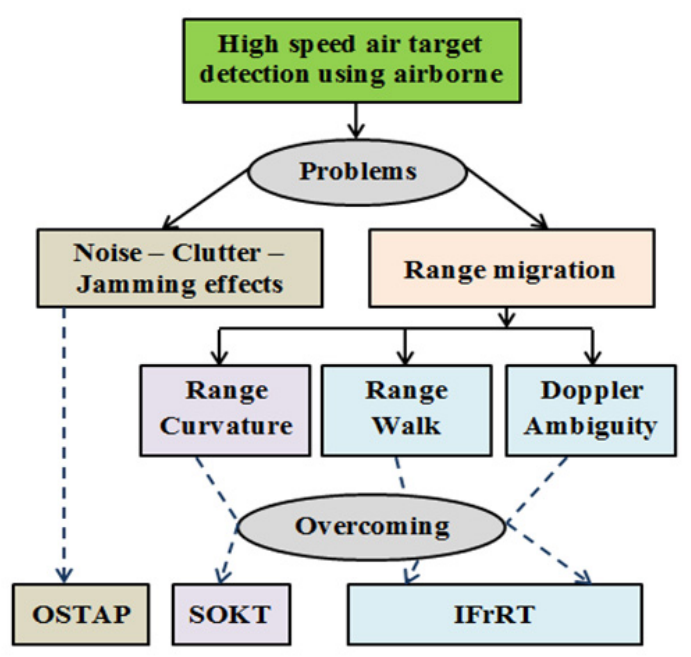

Fig. 1. The problem formulation architecture.

one is how to remove the environmental interferences (noise, clutter, and jamming signals), and the second one is how to make compensation for the range migration and Doppler ambiguity estimation problems. Fig. 1 shows a block diagram for clarifying the statement.

The high speed air target detection is a key problem for airborne radar. The problem is how to compensate for the range migration, and Doppler ambiguity estimation problems in the presence of the midst interferences (clutter, jamming, and noise), in order to improve the probability of detection and the detector performance of HSMDT. We can increase the observation time. However, the signal energy cannot be effectively accumulated since high speed motion induces the large range migration and the range walk and the Doppler frequency shift in long time coherent integration period and acceleration, these problems make it difficult to improve the target energy and the SNR, so the problem of range migration needs to be considered [22].

There are many approaches that have been used to solve these problems, as discussed before. These approaches used some mathematical transformations such as KT, RFT, FrFT, and their combinations, but these methods ignored the presence of clutter and jamming. Other algorithms combined these methods with another one for clutter rejection, but also each method has its own limitations, starting from this point, the author of this work proposed a new hybrid method for high speed air target detection, using OSTAP/SOKT/IFrRT. The following section will discuss the modeling of the airborne radar signal, the clutter, and the jamming model.

\subsection{Airborne Radar Geometry and Signal Modeling}

The geometry of the airborne radar and target platform, the antenna array elements mounted in the side of the radar and in the direction of flight $\left(N_{O}\right.$ is the number of array element, $\mathbf{v}_{\mathbf{T}}$ is the target radial velocity) are shown in Fig. 2. 


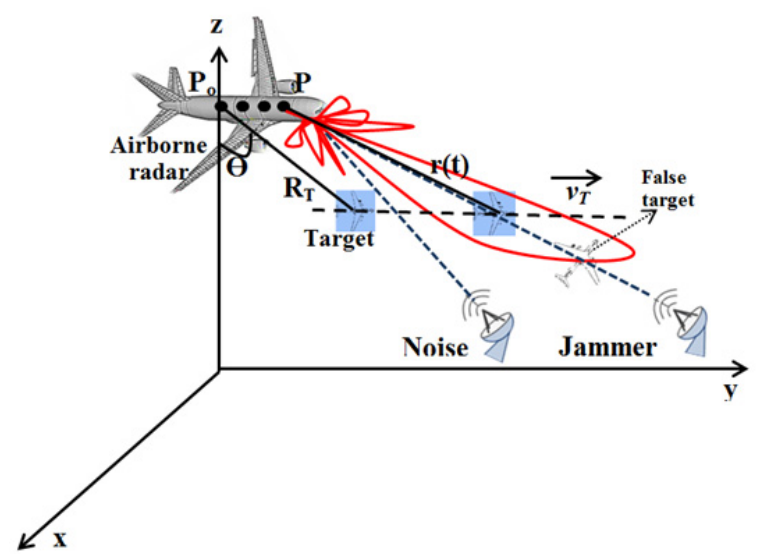

Fig. 2. The geometry of the target and the radar platform.

The data in the testing range bin (referred to as the primary data) is not only comprised of the desired target reflection, but also the returns from unwanted objects (clutter, noise, and jammer). The data can be modeled as [23]

$$
S_{P}=A_{t}+A_{c}+A_{n}+A_{j},
$$

where $S_{P}$ is the total returned primary data, $A_{t}, A_{c}, A_{n}$, and $A_{j}$ denote target, clutter, noise, and problem of range migration. We suppose the velocity of the target $\mathbf{v}_{\mathbf{T}}$ along fixed direction, such as the y-axis direction shown in Fig. 2. So the velocity vector is expressed the scalar. The same is similar other velocity vectors.

In order to achieve a high range resolution, a wide band pulses are used, which are assumed linear frequency modulated (LFM) signals here [24], and $\mathbf{v}_{\mathbf{T}} / c<<1$, where $c$ is the light speed, so the received echo signal $S_{\tau}(t, \tau)$ after down conversion will be in the form.

$$
\begin{aligned}
S_{\tau}(t, \tau)= & \sigma_{t} \operatorname{rect}\left(\frac{\tau-2 r(t) / c}{T_{P}}\right) \cdot \exp \left[j \pi \gamma\left(\tau-\frac{2 r(t)}{c}\right)^{2}\right] \\
& \exp \left[-j \frac{4 \pi r(t)}{\lambda}\right] \cdot \exp \left(-j \frac{4 \pi v_{\mathrm{T}} \tau}{\lambda}\right)
\end{aligned}
$$

where $t=n T(n=0,1, \ldots, N-1)$ is the slow time, $T$ is the pulse repetition time, $c$ is the speed of light, $N$ is the number of coherent integrated pulses, $\tau$ is the fast time, i.e. the range time, $\sigma_{t}$ is the backscattering coefficient of the target, $\operatorname{rect}(x)$ is the window function and equal to one for $|x| \leq(1 / 2)$ or zero if otherwise, $T_{P}$ is the pulse width, $r(t)$ is the instantaneous range between the radar and the target, $\lambda=c / f_{c}$ is the wave length, $f_{c}$ is the carrier frequency, $\gamma$ is the modulation rate. We can neglect the higher-order components, so that the instantaneous range $r(t)$ depicted in Fig. 2 between the radar, and the target can be written as

$$
r(t)=R_{T}+v_{T} \tau+a_{T} t^{2} / 2
$$

where $R_{T}$ is the initial range from the radar platform to the target, and $a_{T}$ is the radial acceleration of the target. The received signal after range compression in the slow timerange frequency $(t-f)$ domain can be expressed as [8]

$$
\begin{aligned}
S_{r}(t, f)=\sigma_{t} \operatorname{rect}\left(\frac{f+f_{d} / 2}{B-f_{d}}\right) \cdot \exp \left[-j \frac{4 \pi\left(f+f_{c}+f_{d}\right.}{c} r(t)\right] \\
\exp \left[-j \frac{2 \pi f}{\gamma} f_{d}\right] \cdot \exp \left(-j \frac{\pi}{\gamma} f_{d}^{2}\right)
\end{aligned}
$$

where $B$ is the signal bandwidth and $f_{d}=2\left|v_{T}\right| / \lambda$ denotes the Doppler frequency. Substituting (3) into (4) yields

$$
\begin{aligned}
S_{r}(t, f)= & \sigma_{t} \operatorname{rect}\left(\frac{f+f_{d} / 2}{B-f_{d}}\right) . \\
& \exp \left[-j \frac{4 \pi\left(f+f_{c}+f_{d}\right)}{c} R_{T}\right] . \\
& \exp \left[-j \frac{4 \pi\left(f+f_{c}+f_{d}\right)}{c} v_{T} t\right] . \\
& \exp \left(-j \frac{2 \pi f}{\gamma} f_{d}\right) \cdot \\
& \exp \left[-j \frac{2 \pi\left(f+f_{c}+f_{d}\right)}{c} a_{T} t^{2}\right] . \\
& \exp \left(-j \frac{\pi}{\gamma} f_{d}^{2}\right)
\end{aligned}
$$

because the high speed targets and the low pulse repetition frequency $P R F$ under sampling would occur. Therefore, the velocity of the target can be expressed as.

$$
v_{T}=\Gamma v_{b}+v_{0}
$$

where $v_{b}$ is the blind velocity, $\Gamma$ is the Doppler center ambiguity number. It is noted that the azimuth Doppler frequency ranges from $(-P R F / 2)$ to $(P R F / 2), v_{b}$ is estimated to be $v_{b}=\lambda P R F / 2$, and $v_{0}<v_{b} / 2$. Substituting (6) into (5) yields

$$
S_{r}(t, f)=S_{1}(t . f) H_{1}(t, f)
$$

where

$$
\begin{aligned}
& S_{1}(t, f)= \sigma_{t} \operatorname{rect}\left(\frac{f+f_{d} / 2}{B-f_{d}}\right) . \\
& \exp \left(-j \frac{\pi f_{d}^{2}}{\gamma}\right) . \\
& \exp \left[-j \frac{4 \pi\left(f+f_{c}+f_{d}\right)}{c} R_{T}\right] . \\
& \exp \left(-j \frac{2 \pi f}{\gamma} f_{d}\right) \cdot \\
& \exp \left[-j \frac{4 \pi\left(f+f_{c}+f_{d}\right)}{c} v_{0} t\right] . \\
& \exp \left[-j \frac{2 \pi\left(f+f_{c}+f_{d}\right)}{c} a_{T} t^{2}\right], \\
& H_{1}(t, f)=\exp \left[-j \frac{4 \pi\left(f+f_{c}+f_{d}\right)}{c} \Gamma v_{b} t\right],
\end{aligned}
$$


supposing that $\xi=\left(f+f_{d}\right) / f_{c}$ and ignoring the effect of $f_{d}$ on $\xi$, so that $\xi$ can be simplified into $\xi \approx f / f_{c}$. Thus, equations (7), (8), and (9) can be rewritten as.

$$
S_{r}(t, f)=S_{1}(t . f) H_{1}(t, f)
$$

where

$$
\begin{aligned}
S_{1}(t, f)= & \sigma_{t} \operatorname{rect}\left(\frac{f+f_{d} / 2}{B-f_{d}}\right) . \\
& \exp \left(-j \frac{\pi f_{d}^{2}}{\gamma}\right) . \\
& \exp \left[-j \frac{4 \pi R_{T}}{\lambda}(1+\xi)\right] . \\
& \exp \left[-j \frac{4 \pi v_{0} t}{\lambda}(1+\xi)\right] . \\
& \exp \left[-j \frac{2 \pi a_{T} t^{2}}{\lambda}(1+\xi)\right] . \\
& \exp \left(-j \frac{2 \pi f}{\gamma} f_{d}\right), \\
& H_{1}(t, f)=\exp \left[-j \frac{4 \pi \Gamma v_{\mathbf{b}} t}{\lambda}(1+\xi)\right] .
\end{aligned}
$$

\subsection{Clutter, Noise, and Jamming Modeling}

Airborne radar system would suffer from ECM scenarios, which results in confusion on detecting and identifying the true target. Methods of existing ECM can be classified as suppressive jamming or deceptive jamming [2], [6]. Here we supposed to generate deceptive jamming, in which the false targets are usually formed by the digital radio frequency memory (DRFM) which generate many times delayed and Doppler modulated radar signal replicas to deceive the radar system. Thus, the deceptive jamming $S_{J}$ can be expressed as [23]

$$
S_{J}=\sum_{P=1}^{P_{2}} \sum_{i=1}^{N_{P}} \xi_{P, i} \mathbf{a}_{J v}\left(r_{P}, \theta_{P}\right) \otimes \mathbf{a}_{J u}\left(\theta_{P}\right)
$$

where $P_{2}$ is the number of the generated false targets, $N_{P}$ indicates the number of false targets generated by the $p^{\text {th }}$ DRFM, $\xi_{P, i}$ is the corresponding amplitude, $r_{P}$ and $\theta_{P}$ are the range and angle parameters of the $p^{\text {th }}$ DRFM, $\mathbf{a}_{J v}\left(r_{P}, \theta_{P}\right)$, and $\mathbf{a}_{J u}\left(\theta_{P}\right)$, are the jamming temporal (fast time - slow time) steering vector, and the jamming spatial steering vector respectively. Note that the false targets are time delayed and Doppler modulated, thus they can be at any range bin and any Doppler cell. We assumed that the jammer source will send one false target as we will see in the simulation part, its component in the received signal can be modeled as

$$
A_{J}=\sigma^{j} \mathbf{v}^{\mathrm{j}}\left(\varphi_{j}, \theta_{j}, f_{D}^{j}, l\right)
$$

where $\sigma^{j}$ is the complex radar cross section (RCS) of the jamming, $\mathbf{v}^{\mathrm{j}}\left(\varphi_{j}, \theta_{j}, f_{D}^{j}, l\right)$ is a snapshot vector of jamming- plus-noise. The noise is modeled as complex white Gaussian with variance $\sigma_{n}{ }^{2}$. The clutter can be expressed as [23]

$$
S_{C}=\sum_{i=1}^{N_{C}} \xi_{i} \mathbf{a}_{C v}\left(r, \theta_{i}\right) \otimes \mathbf{a}_{C u}\left(\theta_{i}\right)
$$

where $N_{C}$ is the statistically independent clutter patches, $\xi_{i}$ is the scattering coefficient of the $i^{\text {th }}$ patch, and $r, \theta_{i}$ are the range and angle parameters of the $i^{\text {th }}$ patch, so the clutter signal can be modeled as.

$$
A_{C}=\sigma^{c} \mathbf{v}^{\mathbf{c}}\left(\varphi_{c}, \theta_{c}, f_{D}^{c}, l\right)
$$

where $\sigma^{c}$ is the complex RCS of the clutter, $\mathbf{v}^{\mathbf{C}}\left(\varphi_{c}, \theta_{c}, f_{D}^{c}, l\right)$ is snapshot vector of clutter-plus-noise, The Doppler frequency of clutter and jamming are $f_{D}^{c}, f_{D}^{j}$.

\section{OSTAP Algorithm}

Reduced rank STAP approaches utilizing data dependent transformations, such as the principal components inverse algorithm [25], Eigen canceller algorithm [26], [27], cross-spectral metric (CSM) [28] and multistage Wiener filter (MWF) [29]. Both the CSM and MWF all have high performances and low sampling requirements. However, the detection performance degrades rapidly as the number of eigenvectors retained is decreased below the rank of clutter. The penalty for underestimating the rank of the total noise contribution can be great [7]. The most of aforementioned literatures published on the STAP are concerned about how to reduce the number of the secondary data, how to enhance the capability of detecting the slowly moving target, recently [30] proposed a model of STAP for anti-jamming and clutter rejection and so on. Even though, the problem of range migration compensation is few discussed [2].

Fig. 3 shows the construction of OSTAP and explains the principle of removing clutter and jamming, here the pre-filter is used to attenuate strong clutter before it can be modulated, then it passes through the side lobes of a jamming cancellation beam. Pre-filtering seeks to attenuate such clutter before the modulation occurs. It is assumed that the airborne radar system is a uniform linear array composed of $N_{O}$ elements with element spacing $d$, and the array is placed along the flight direction. The radar transmits $M$ pulses in each CPI, $x_{n m}$ is the complex sample from the $n^{\text {th }}$ element at the $m^{\text {th }}$ pulse [18]. The received data for each range bin may be organized into an $N \times M$ matrix.

$$
\mathbf{S}=\left[\begin{array}{cccc}
\mathbf{s}_{11} & \mathbf{s}_{12} & \ldots & \mathbf{s}_{1 M} \\
\mathbf{s}_{21} & \mathbf{s}_{12} & \ldots & \mathbf{s}_{21} \\
\vdots & \vdots & \ldots & \vdots \\
\mathbf{s}_{N 1} & \mathbf{s}_{N 2} & \ldots & \mathbf{s}_{N M}
\end{array}\right]
$$

The data matrix $\mathbf{S}$ is expressed as an $N M \times I$ form by stacking the columns of $\mathbf{S}$. These arrays or vectors are 


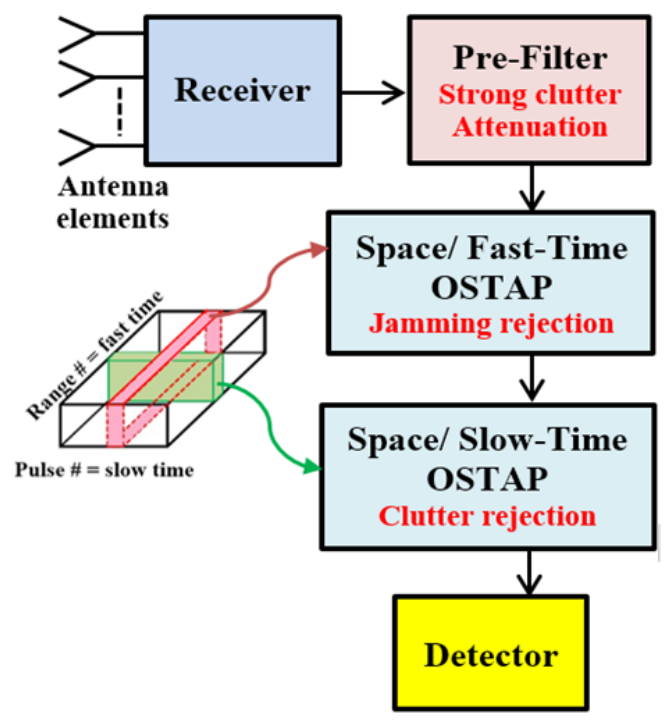

Fig. 3. The OSTAP construction for clutter and jamming cancellation.

referred as "snapshot" or "samples." The optimum STAP processor (OSTAP) computes weights associated with the optimum weighted linear combination of the elements of sample vectors to determine if a hypothetical target is present or not. It is noted that the calculation of the optimum weight vector needs to calculate the $N M \times N M$ space-time interference sample correlation matrix $\mathbf{R}$ and its inverse [18].

Assuming that one target is present in a single range bin. Consequently, the detection range bin data, usually referred to as the primary data, components $S_{p}, A_{c}$ and $A_{n}$ are the space-time snapshot of the target, the clutter and the noise, respectively. The space-time clutter echoes from a cell in a given range are a superposition of the returns from all the stationary scatterers within a cell [18], so the primary data $S_{p}$ in (1) can be written as

$$
\begin{gathered}
S_{p}=b \mathbf{a}\left(u_{t}, \mathbf{v}_{\mathbf{T}}\right)+\sigma^{c} \mathbf{v}^{\mathbf{c}}\left(\varphi_{c}, \theta_{c}, f_{D}^{c}, l\right)+ \\
\sigma_{n}^{2}+\sigma^{j} \mathbf{v}^{\mathbf{j}}\left(\varphi_{j}, \theta_{j}, f_{D}^{j}, l\right)
\end{gathered}
$$

where $b$ is the target complex amplitude, $\mathbf{a}\left(u_{t}, \mathbf{v}_{\mathbf{T}}\right)$ is the space-time steering vector of the target with the normalized spatial and temporal frequencies $u_{t}=2 \pi d \cos \theta_{t} / \lambda$ and $\mathbf{v}_{\mathbf{T}}=2 \pi f_{d} / f_{r}$, respectively, $\lambda$ is the wavelength, $f_{r}$ is the pulse repetition frequency, $\theta_{t}$ is the target azimuth, the steering vector $\mathbf{a}(u, \mathbf{v})$ can be given by.

$$
\mathbf{a}(u, \mathbf{v})=\mathbf{a}(\mathbf{v}) \otimes \mathbf{a}(u)
$$

where $\mathbf{a}(\mathbf{v})$ is the temporal steering vector with size of $M \times 1, \mathbf{a}(\mathbf{v})=\left[1 \mathrm{e}^{-j \mathbf{v}} \ldots \mathrm{e}^{-j(M-1) \mathbf{v}}\right]^{T}, \mathbf{a}(u)$ is the spatial steering vector with size of $N \times 1, \mathbf{a}(u)=\left[1 \mathrm{e}^{-j u} \ldots \mathrm{e}^{-j(N-1) u}\right]^{T}$, and $(.)^{T}$ is the transpose, and $\otimes$ is the Kronecker product. Similarly to [18], the data in the reference bin, which is referred to as the secondary data, can be written as

$$
S_{p}=\sigma^{c} \mathbf{v}^{\mathbf{c}}\left(\varphi_{c}, \theta_{c}, f_{D}^{c}, l\right)+\sigma_{n}^{2}+\sigma^{j} \mathbf{v}^{\mathbf{j}}\left(\varphi_{j}, \theta_{j}, f_{D}^{j}, l\right)
$$

The optimum-adaptive processor (OAP) of the STAP is given by [1]

$$
W_{s}=\mu R^{-1} \mathbf{a}\left(u_{t}, \mathbf{v}_{\mathbf{T}}\right)
$$

where $\mu$ is a constant scalar, $\mathbf{R}$ is the space-time covariance matrix of the interference, which is related the clutter, the jammer, and the noise [1], it can be written as

$$
\mathbf{R}=\mathbf{R}_{\mathbf{C}}+\mathbf{R}_{\mathbf{J}}+\sigma^{2} I
$$

where $\mathbf{R}_{\mathbf{C}}, \mathbf{R}_{\mathbf{J}}$ are the clutter and jammer covariance matrices respectively, and $\sigma^{2} I$ denotes the noise. Due to the range migration and range walk problems of the HSMDT the primary data in equation (18) can be modeled as

$$
\tilde{X}_{p}=b \tilde{\mathbf{a}}\left(u_{t}, v_{t}\right)+\sigma^{c} \mathbf{v}^{\mathbf{c}}\left(\varphi_{c}, \theta_{c}, f_{D}^{c}, l\right)+\sigma_{n}{ }^{2} \sigma^{j} \mathbf{v}^{\mathrm{j}}\left(\varphi_{j}, \theta_{j}, f_{D}^{j}, l\right)
$$

where $\tilde{X}_{p}$ is the primary data with range migration, $\tilde{\mathbf{a}}\left(u_{t}, \mathbf{v}_{\mathbf{T}}\right)$ is the steering vector of the target in the presence of range walk and range migration, and we assume for simplicity that the clutter and the jammer have no range walk and range migration. This will be valid when the airborne radar platform moves with not very high speed. The steering vector in case of range walk and range migration can be given as

$$
\tilde{\mathbf{a}}\left(u_{t}, \mathbf{v}_{\mathbf{T}}\right)=\tilde{\mathbf{a}}(\mathbf{v}) \otimes \mathbf{a}(u)
$$

where $\tilde{\mathbf{a}}(\mathbf{v})=\left[\begin{array}{lll}\xi_{0} \xi_{1} e^{-j v} & \ldots & \xi_{M-1} e^{-j(M-1) v}\end{array}\right]^{T}$ is the new temporal steering vector, $\xi$ is a scalar representing the effect of the range migration and range walk on the $m^{\text {th }}$ pulse.

In this case, the signal match terms $\mathbf{a}\left(u_{t}, \mathbf{v}_{\mathbf{T}}\right)$ in the weight vector $\mathbf{W}_{\mathbf{S}}$ can't perform its function well, so the compensation of the range walk and the range migration must be considered. The performance of the OSTAP is closely related with the clutter properties, whereas the direct use of the compensation methods will affect the clutter distribution which in turn affects the performance of the OSTAP. So we must first apply OSTAP to remove the interference of the clutter, the jammer, and the noise from the echo signal. The eigenvalue decomposition of $R$ can be expressed as follows

$$
\mathbf{R}=\sum_{l=1}^{N M} \lambda_{l} u_{l} u_{l}{ }^{H}=\sum_{l=1}^{Q} \lambda_{l} u_{l} u_{l}{ }^{H}+\sigma^{2} \sum_{l=Q+1}^{N M} u_{l} u_{l}{ }^{H} .
$$

The eigenvalues $\lambda_{l}(l=1,2, \ldots, Q)$ come from the clutter and the jamming, and the rest $(N M-Q)$ eigenvalues come from the noise component, $(.)^{H}$ is the conjugate transpose. The eigenvectors $u_{l}(l=1,2, \ldots \ldots, Q)$ are corresponding to the clutter eigenvalues span the clutter subspace. The projection matrix orthogonal to the clutter subspace can be written as

$$
\mathbf{P}_{\mathbf{c}}{ }^{\perp}=1-\sum_{l=1}^{Q} u_{l} u_{l}^{H} .
$$

Now it is easy to verify that $\lim _{n \rightarrow \infty} \sigma^{2 n} \mathbf{R}^{-\mathbf{n}}=\mathbf{P}_{\mathbf{c}}{ }^{\perp}$. 
If the eigenvalues $\lambda_{1} \gg \sigma^{2}(l=1,2, \ldots \ldots, Q)$, and the subspace $\mathbf{P}_{\mathbf{c}}{ }^{\perp}=\mathbf{R}^{-1}, \mathbf{R}^{-1}$ is used as an approximation of $\mathbf{P}_{\mathbf{c}}{ }^{\perp}$ to suppress the clutter and the jamming and the noise in the received data, it is easy to calculate and to avoid the order (Q) selection of $\mathbf{P}_{\mathbf{c}}{ }^{\perp}$. In practice, the matrix $\mathbf{R}$ is not known a priori and must be estimated, and the estimated covariance matrix is denoted as $\widehat{\mathbf{R}}$.

\section{Parameters Estimation Algorithms}

Using the OSTAP method to reject the clutter, the jamming, and the noise, the target range can be well extracted, but the problem of range migration and the Doppler ambiguity phenomenon occurs. In this section, we introduce a modified method, the hybrid of the SOKT and the IFrRT methods, to deal with these problems. From (3), it has observed that the range migration involves two components, namely, the range walk and range curvature. Moreover the third term that introduces the acceleration and Doppler ambiguity is involved. If one wants to accurately calculate a range of a target, the range migration should be well corrected. However, in practice, the range migration is always difficult to be accurately compensated [22], and the acceleration effect must be also compensated.

\subsection{Range Curvature Correction using SOKT}

In high signal to clutter-jamming-noise ratio (SCJNR) situations, the motion parameters can be estimated based on the Doppler analysis and used for envelope alignment. However, in the low SNR situation, it is difficult to detect the motion parameters directly from the received signals. Keystone transform (KT) [19], [14] can be applied for the envelope alignment. The SOKT corrects the range curvature and the range walk by making compensation of a quadratic phase term.

The SOKT, which performs scaling $t=\left[f_{c} / f+f_{c}\right]^{1 / 2} t_{a}$ in the $(t-f)$ domain, can be applied for the range curvature correction. Also the SOKT is a process of resampling. It consists of two steps [19]. First, the reconstruction of the continuous signal from the sampled version, and the second is the sampling of the reconstructed signal at the new sampling grid. By substituting the scaling formula into (11) we obtain

$$
\begin{aligned}
& S_{1}^{\prime}\left(t_{a}, f\right)=\sigma_{t} \operatorname{rect}\left(\frac{f+f_{d} / 2}{B-f_{d}}\right) \exp \left(-j \frac{\pi f_{d}^{2}}{\gamma}\right) \\
& \exp \left[-j \frac{4 \pi R_{T}}{\lambda}(1+\xi)\right] \cdot \exp \left[-j \frac{4 \pi \mathbf{v}_{0} t_{a}}{\lambda}\left(\frac{f+f_{c}}{f_{c}}\right)^{1 / 2}\right] . \\
& \exp \left[-j \frac{4 \pi \mathbf{v}_{0} t_{a}}{\lambda}\left(\frac{f+f_{c}}{f_{c}}\right)^{1 / 2}\right] . \\
& \exp \left[-j \frac{2 \pi a_{T} t_{a}^{2}}{\lambda}\right] \cdot \exp \left(-j \frac{2 \pi f}{\gamma} f_{d}\right)
\end{aligned}
$$

then taking the first-order Taylor series expansion over $\left(\frac{f+f_{c}}{f_{c}}\right)^{1 / 2}$, it has found that.

$$
\begin{aligned}
S_{1}^{\prime}\left(t_{a}, f\right) \approx & \sigma_{t} \operatorname{rect}\left(\frac{f+f_{d} / 2}{B-f_{d}}\right) \cdot \exp \left(-j \frac{\pi f_{d}^{2}}{\gamma}\right) . \\
& \exp \left[-j \frac{4 \pi R_{T}}{\lambda}(1+\xi)\right] \cdot \\
& \exp \left[-j \frac{4 \pi \mathbf{v}_{0} t_{a}}{\lambda}\right] \cdot \exp \left[-j \frac{2 \pi \mathbf{v}_{0} t_{a}}{c}\right] . \\
& \exp \left[-j \frac{2 \pi a_{T} t_{a}^{2}}{\lambda}\right] \cdot \exp \left(-j \frac{2 \pi f}{\gamma} f_{d}\right) .
\end{aligned}
$$

Since $H_{1}(t, f) \approx H_{1}\left(t_{a}, f\right)$ so equation (10) can be rewritten as

$$
\begin{aligned}
S_{r}^{\prime}\left(t_{a}, f\right)= & S_{1}^{\prime}\left(t_{a} \cdot f\right) H_{1}\left(t_{a}, f\right) \\
& \approx \sigma_{t} \operatorname{rect}\left(\frac{f+f_{d} / 2}{B-f_{d}}\right) . \\
& \exp \left(-j \frac{\pi f_{d}^{2}}{\gamma}\right) \exp \left[-j \frac{4 \pi R_{T}}{\lambda}\left(1+\frac{f}{f_{\mathrm{c}}}\right)\right] . \\
& \exp \left[-j \frac{4 \pi \mathbf{v}_{\mathbf{T}} t_{a}}{\lambda}\right] \exp \left[-j \frac{2 \pi a_{T} t_{a}^{2}}{\lambda}\right] . \\
& \exp \left[-j \frac{4 \pi f}{c}\left(\frac{v_{0}}{2}+\Gamma \mathbf{v}_{\mathbf{b}}\right) t_{a}\right] . \\
& \exp \left(-j \frac{2 \pi f}{\gamma} f_{d}\right) .
\end{aligned}
$$

From (30), it has been found that the second-order couplings effect (range curvature) QRCM has been removed. However, residual coupling (RCM) determined by the ambiguity number, and the Doppler frequency migration (DFM) are still present [8], which corresponds to the range walk and the Doppler Ambiguity estimation and the acceleration. The instantaneous range between the radar and the target can now be written as

$$
r(t)=R_{T}+a_{T} t^{2} / 2 \text {. }
$$

We can see from (31) that the term of range migration $\left(\mathbf{v}_{\mathbf{T}} \tau\right)$ (the range curvature and the range walk) is removed using the SOKT but not completely, it still has a little component of a range walk, $\left[\left(\mathbf{v}_{0} / 2+\Gamma \mathbf{v}_{\mathrm{b}}\right) t_{a}\right]$ in (30).

\subsection{Range Walk Correction and Doppler Ambiguity Estimation using IFrRT}

Although the range curvature correction and the quadric phase term compensation have been performed, the accurate estimation of parameters cannot be obtained due to the residual range walk caused by integral multiples of the blind velocity and half of the unambiguous velocity [8]. The RFT is an effective way to measure, which adopted a small and discrete number of slopes exists in the $(r, \tau)$ do- 
main, the slope of a straight-line [20]. Since the RFT only uses the amplitude information, its result can be impacted by the low SNR.

The Fraction Radon Fourier Transform (FrRFT) is used to realize long-time coherent integration for the HSMDT. Comparing to the RFT, the FrRFT can be greatly extended to the range curvature and the Doppler frequency migration effect in the integration time, and restricted by minimizing coherent integration gain and dwell of an antenna beam. From (30), there is a second-order phase term of the slow time that can adversely influence the coherent accumulation of the signal, so the dechirping operation [20] is used to remove the influence and express as

$$
\begin{aligned}
& \operatorname{IFrRT}\left(A_{m}, \text { target velocity : } S_{r}^{\prime}\left(t_{a}, f\right)\right)= \\
& \qquad \int_{t_{a}} S_{r}^{\prime}\left(s, t_{a}\right) \cdot \exp \left[-j \frac{2 \pi a_{T} t_{a}^{2}}{\lambda R_{T}}\right] d t_{a}
\end{aligned}
$$

where $A_{m}$ is the ambiguity number, $s=\left[2 R_{2 T}\left(t_{a}\right)\right] / c$, so,

$$
R_{2 T}(\mathbf{v})=R_{T}+A_{m} \frac{f}{2} t_{a} .
$$

Equation (29) is the same as the definition of the FrRFT, called the IFrRT. The difference between FrRFT and IFrRT is of function expressed as $s=f\left(t_{a}\right)$, and it doesn't have as straight line. The estimation error in the baseband velocity could lead to an unsatisfying result by (31) and the expression in (32) must be modified to solve this problem. Substituting (30) into (32) and ignoring unimportant phase terms [20], we obtain

$$
\begin{aligned}
& \operatorname{IFrRT}\left(A_{m}, \text { target velocity : } S_{r}^{\prime}\left(t_{a}, f\right)\right) \simeq \\
& \int_{t_{a}} \operatorname{sinc}\left[B\left(s-\frac{2 R_{2 T}\left(t_{a}\right)}{c}\right)\right] \cdot \exp \left[-j 2 \pi \frac{a_{T}{ }^{2}-a_{T}^{\prime 2}}{\lambda R_{T}}-t_{a}{ }^{2}\right] d t_{a} .
\end{aligned}
$$

The phase terms in integral become zero. Thus, a coherent accumulation is realized, and the IFrRT can now work well at low SNR condition. So that the acceleration

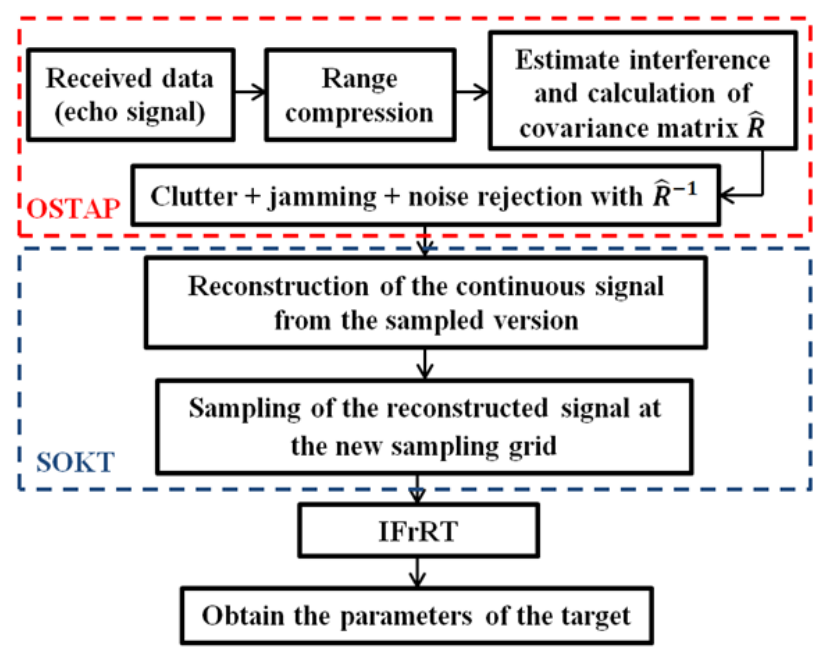

Fig. 4. The flow chart of the proposed algorithm. and the Doppler ambiguity were compensated using IFrRT. The instantaneous range between the radar and the target can now be written as

$$
r(t)=R_{T}
$$

From (35), it has found that the term of range migration $\left(\mathbf{v}_{\mathbf{T}} \tau\right)$ (the range curvature and the range walk) is compensated completely using the IFrRT and the acceleration and the Doppler ambiguity $\left(a_{T} t^{2} / 2\right)$ was also compensated.

The flow chart shown in Fig. 4 describes the proposed algorithm. The detailed steps are as follows:

Step 1: Implement the range compression for the received data;

Step 2: Applying the OSTAP by estimating the space-time interference covariance matrix to be approximated to suppress the clutter and the jamming and the noise from the received space time data;

Step 3: Applying the SOKT by reconstructing of the continuous signal from the sampled version and the sampling to reconstruct a new signal at the new sampling grid in order to implement range migration compensation;

Step 4: Applying the IFrRT to compensate the acceleration and the Doppler ambiguity estimation compensation;

Step 5: Calculating the real range for target after the interference rejection and the range estimation compensation.

\subsection{Performance Improvement and Detection Probability}

From probability theory, the probability of detection for a single radar can be calculated by [22]

$$
P_{d}=\int_{T}^{\infty} P(v) d v
$$

where $P(v)$ is the probability density of the power of IF target signal plus noise, and $T$ is the threshold level for the cases of swerling I-II targets with individual pulse detection, $P(v)$ is [31]

$$
P(v)=\frac{1}{1+S C J N R} \exp \left[\frac{-\mathbf{v}}{1+S C J N R}\right],
$$

so detection probability $P_{d}$ of the target is

$$
P_{d}=\exp \left[\frac{-T}{1+S C J N R}\right] \text {. }
$$

The signal-to-clutter-plus-jamming-plus-noise ratio (SCJNR) is commonly used to assess the detection performance of airborne radar systems. SCJNR is defined as the ratio of clutter-plus-jamming-limited output $S C J N R$ to the noise-limited output SNR [23]. 


$$
S C J N R=\frac{S C J N R_{\text {out }}}{S N R_{\text {out }}}=\frac{\mathbf{W}^{\mathbf{H}} \mathbf{R}_{\mathbf{t}} \mathbf{W}_{\mathbf{s}}}{\mathbf{W}^{\mathbf{H}}{ }_{\mathbf{s}}\left(\mathbf{R}_{\mathbf{C}}+\mathbf{R}_{\mathbf{J}}+\mathbf{R}_{\mathbf{n}}\right) \mathbf{W}_{\mathbf{s}}} \frac{\sigma_{n}{ }^{2}}{\sigma_{t}^{2} N K}
$$

where $\mathbf{R}_{\mathbf{t}}$ is the target covariance matrix, $\mathbf{W}^{\mathbf{H}}$ is the conjugate transpose operator, and $\sigma_{t}{ }^{2}, \sigma_{n}{ }^{2}$ are the power of the true target and the noise. By calculating the probability of detection for the radar receiver in case of using the proposed algorithm (OSTAP/SOKT/IFrRT) and comparing these results with the probability of detection for the same system but in case of using the tradition techniques (without the STAP - with only the SOKT - with the IFrFT - with only the RFT), it has been found that the probability of detection for the proposed algorithm is higher than the other models.

\section{Simulation and Results}

The parameters used in simulation are listed in Tab. 1.

\begin{tabular}{|c|c|}
\hline Parameter & Value \\
\hline Carrier frequency $(\mathrm{GHz})$ & 10 \\
\hline Pulse width $(\mu \mathrm{s})$ & 3 \\
\hline Bandwidth $(\mathrm{MHz})$ & 100 \\
\hline Sampling rate $(\mathrm{MHz})$ & 220 \\
\hline Pulse repetition frequency $(\mathrm{KHz})$ & 800 \\
\hline Number of integrated pulses $($ pulse) & 512 \\
\hline Platform velocity $(\mathrm{m} / \mathrm{s})$ & 150 \\
\hline Jammer to noise ratio $(\mathrm{dB})$ & 34 \\
\hline Clutter to noise ratio $(\mathrm{dB})$ & 34 \\
\hline Noise figure $(\mathrm{dB})$ & 4.5 \\
\hline Main antenna gain $(\mathrm{dB})$ & 20 \\
\hline side antenna gain $(\mathrm{dB})$ & 20 \\
\hline
\end{tabular}

Tab. 1. Simulation parameters.

In order to remove the interference (clutter, jamming, and noise) from the received data, the OSTAP method has been used first. Fig. 5 shows the target echo signal before applying the OSTAP. The target is at the range $95 \mathrm{~km}$, the jamming signal (false target) at range $160 \mathrm{~km}$, and the clutter signal power are high, also we can see clearly the Doppler ambiguity and range migration problems.

Fig. 6 shows the target echo signal after applying the OSTAP, the target range is $95 \mathrm{~km}$. The jamming signal (false target) is reduced, and the clutter was rejected after applying the OSTAP, but the range migration and acceleration and Doppler ambiguity are still present.

Fig. 7 shows the target echo in three-dimensional 3D graph after applying the OSTAP. It has been found that the jamming signal and the clutter have been reduced that will cause an increase for the SNR, but the range migration and the Doppler ambiguity problems are still existed. In order to get high range accuracy measurement, the new compensation technology needs to be considered.

Next, we apply the SOKT to remove part of the range migration (range curvature) and part of the range walk, and

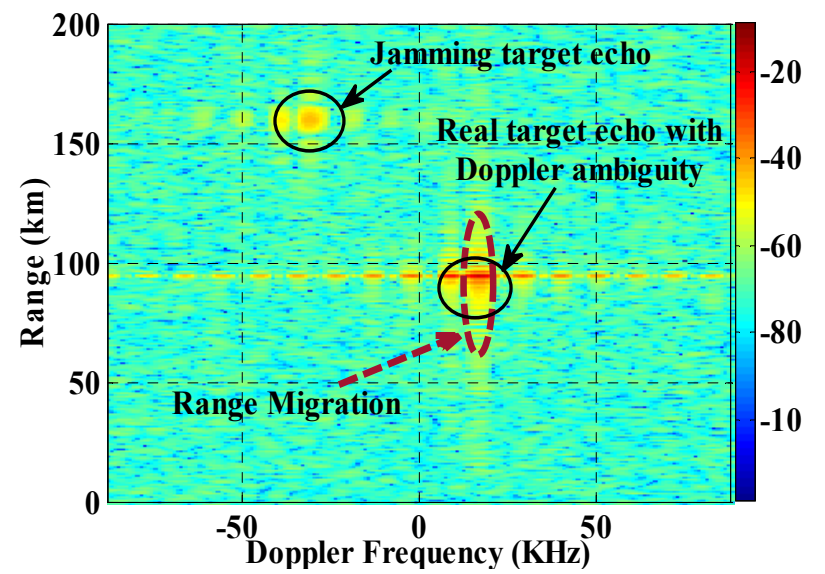

Fig. 5. Received echo of the target before applying the OSTAP.

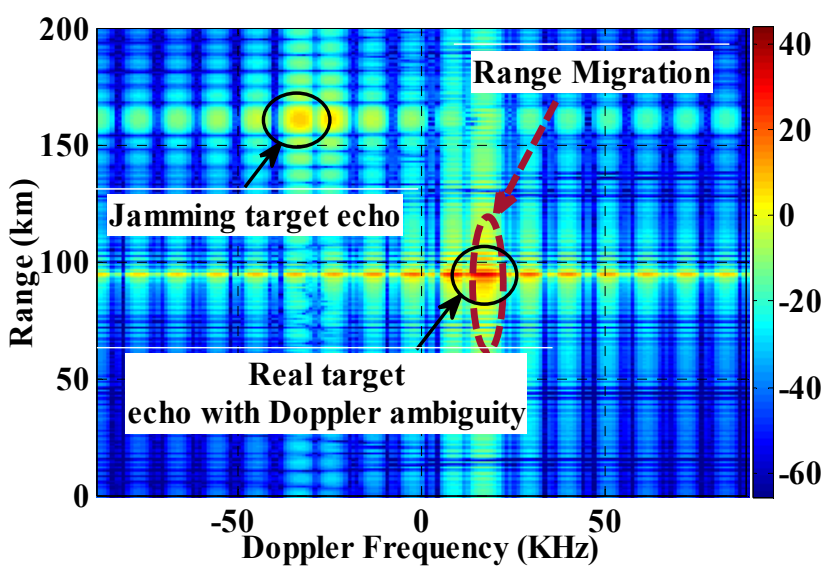

Fig. 6. Received echo of the target after applying the OSTAP.

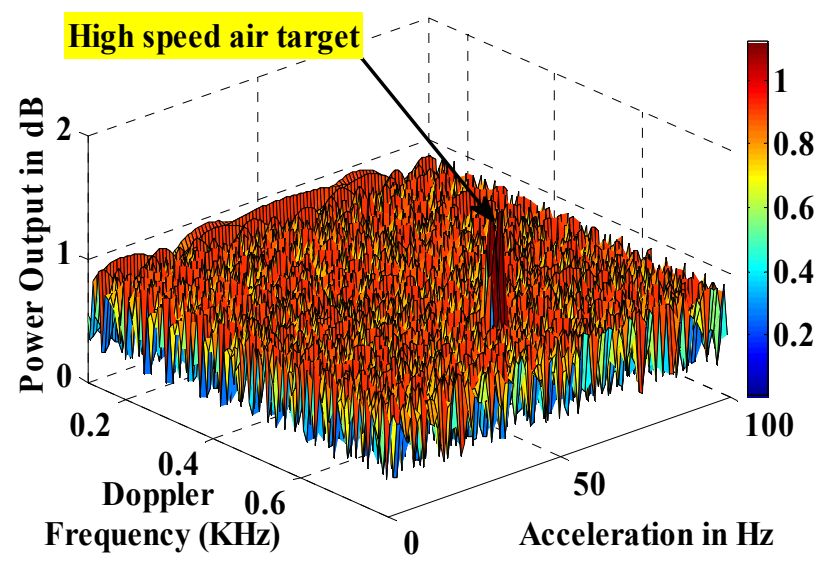

Fig. 7. Target echo after applying the OSTAP in $3 \mathrm{D}$.

then we apply the IFrRT to eliminate the residual range walk and acceleration effect to overcome the Doppler ambiguity.

The results of range compression and after interference rejections are compared in Fig. 8. It can be seen that if the SOKT is not performed so that the range of the target jumps from range bin to another one due to range migration problem (QRCM) that adds unwanted components to the target real range, as shown in Fig. 8 (a). But after applying SOKT the second-order coupling effect (range curvature) (QRCM) has been removed so that the target range was 
detected correctly and stays in the same range bin as shown in Fig. 8 (b). However, residual couplings (RCM) caused by the ambiguity number and DFM are still present because of the range walk.

Fig. 9 displays the received signal after applying the proposed algorithm (Hybrid OSTAP/SOKT/IFrRT). It has been noticed that the interference effect has been reduced and there are no range migration problems and the target is in the true position. Fig. 10 shows the same results in 3D. It shows more clarification to the effect of applying the proposed algorithm.

Fig. 11 is the comparisons of the probability of detection for the radar receiver in case of using the proposed algorithm with that of using the tradition techniques (without STAP-RFT [10]-SOKT/FrFT/RFT [19]-SOKT/IFrRT [20]). It has been found that for the same probability of detection (0.9), the required SNR for the proposed algorithm is the lowest one compared with the other methods.

Fig. 12 compares the SCJNR performance of the proposed method with the other methods as a relation with Doppler frequency. In the proposed model, the interferences can be effectively suppressed, and the target detection performance is well maintained. However, in the traditional methods, the performance degrades dramatically.
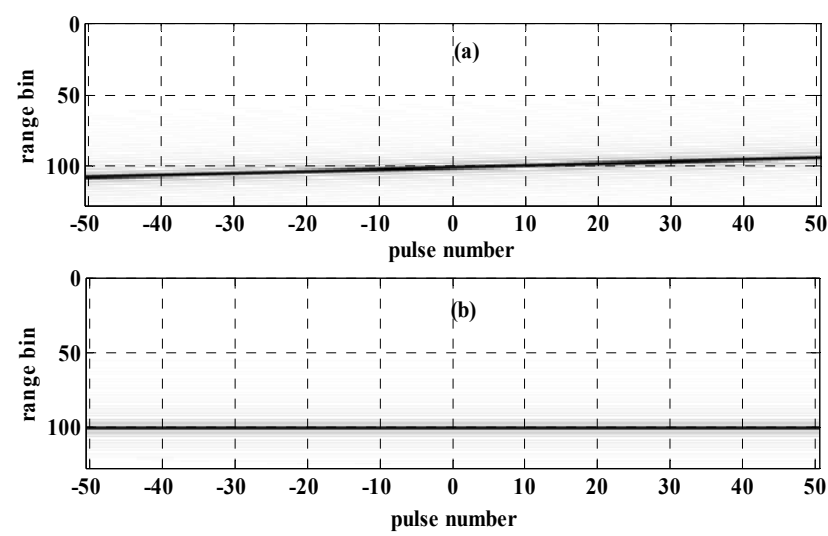

Fig. 8. The range bin: a) before SOKT, b) after SOKT.

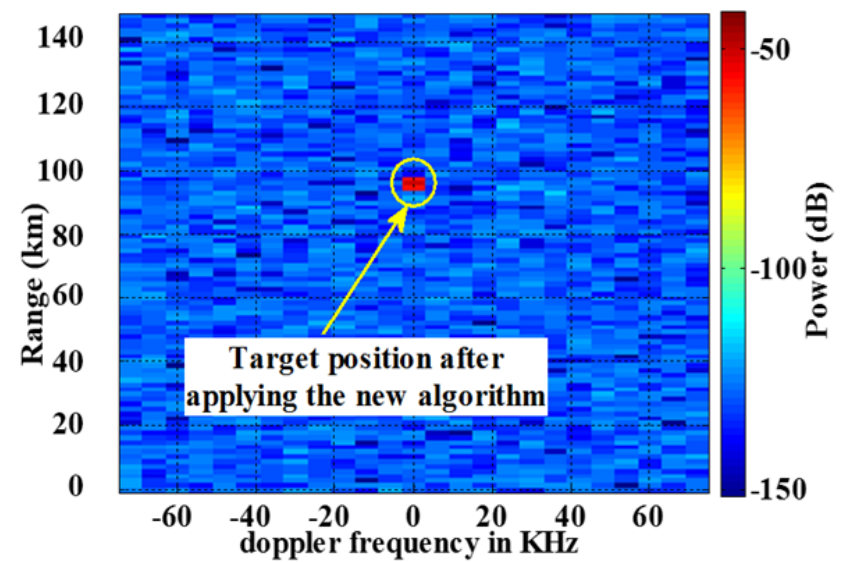

Fig. 9. Received echo of the target after applying the new algorithm (hybrid OSTAP/SOKT/IFrRT).

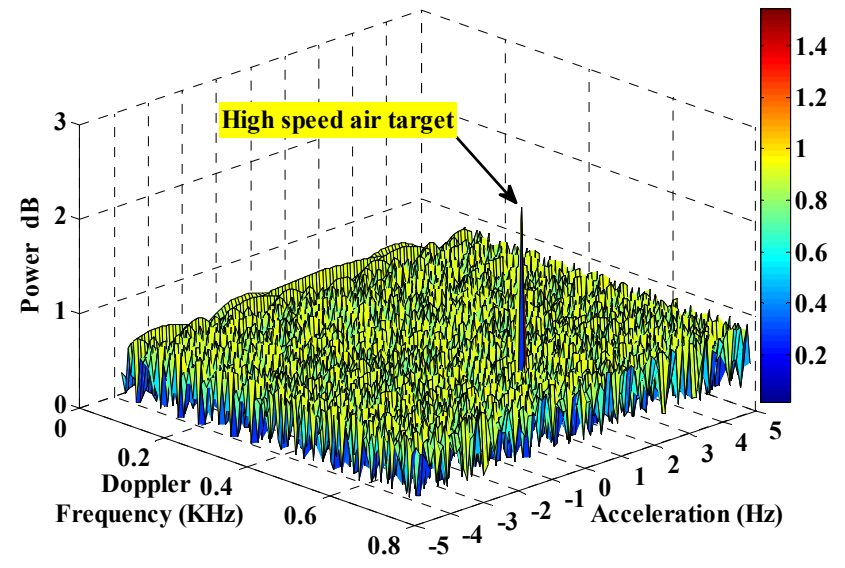

Fig. 10. Received echo of the target after applying the new algorithm (hybrid OSTAP/SOKT/IFrRT) in 3D.

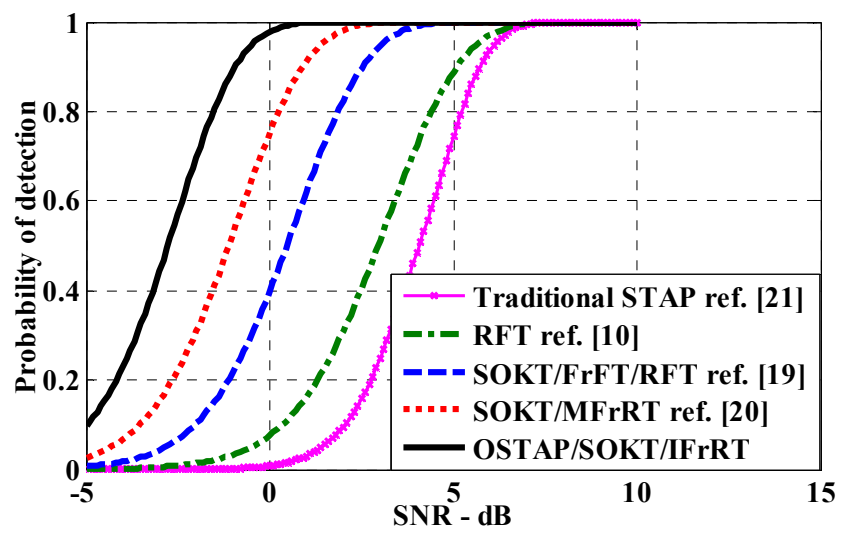

Fig. 11. Probability of detection of the proposed algorithm compared with the old methods.

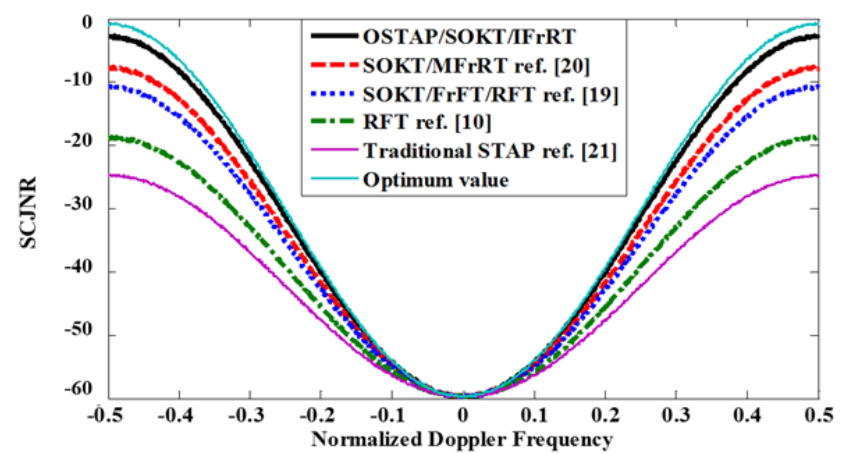

Fig. 12. SCJNR performance.

\section{Conclusion}

This paper has proposed a new method for HSMDT detection in the presence of interference by using airborne radar. The jamming and high clutter effect was added to meet the actual environment, the OSTAP was used to suppress clutter and jamming, the range migration (the range curvature and the range walk), and the Doppler frequency ambiguity estimation problems were completely compensated using SOKT/IFrRT. The simulation results showed that the SNR was improved using OSTAP after clutter and 
jamming suppression, the performance of SCJNR was also well enhanced. The new technique is valid for applying in the monostatic airborne radar application for detecting high speed air targets as well as the ground moving targets.

\section{Acknowledgments}

This work was supported in parts by the National Natural Science Foundation of China (no. 61301211) and the Key Laboratory of Radar Imaging and Microwave Photonics, Ministry of Education, Nanjing University of Aeronautics and Astronautics, Nanjing, 210016, China.

\section{References}

[1] BRENNAN, L. E., REED, I. S. Theory of adaptive radar. IEEE Transaction on Aerospace Electronic Systems, 1973, vol. 9, no. 2 , p. 237-251. DOI: 10.1109/TAES.1973.309792

[2] WEI, Z., ZISHU, H., JUN, L., HONGMIN, L. Multiple-input multiple-output radar multistage multiple-beam beam space reduced-dimension space-time adaptive processing. IET Radar, Sonar and Navigation, 2013, vol.7, no. 3, p. 295-303. DOI: 10.1049 /iet-rsn.2012.0078

[3] WANG, Y.L., CHEN, J.W., BAO, Z., PENG, Y.N. Robust spacetime adaptive processing for airborne radar in non-homogeneous clutter environments. IEEE Transaction on Antenna and Propagation, 2003, vol. 39, no. 1, p. 70-81. DOI: $10.1109 /$ TAES.2003.1188894

[4] KIRSTEINS, I. P., TUFTS, D. W. Adaptive detection using a low rank approximation to a data matrix. IEEE Transaction on Aerospace Electronic Systems, 1994, vol. 30, no. 1, p. 55-67. DOI: $10.1109 / 7.250406$

[5] DIPIETO, R. C. Extended factored space-time processing technique for airborne radar. In Proc. 25th Asilomar Conference on Signals, Systems and Computers. Pacific Grove (CA, USA), October, 1992, vol. 1, p. 425-430. DOI: 10.1109/ACSSC.1992.269236

[6] WU, D., ZHU, D., SHEN, M., ZHU, Z. Time-varying space-time autoregressive filtering algorithm for space-time adaptive processing. IET Radar, Sonar \& Navigation, 2012, vol. 6, no. 4, p. 213-221. DOI: 10.1049/iet-rsn.2011.0095

[7] GUOQING, L., JIAN, L. Moving target detection via airborne HRR phased array radar. IEEE Transactions on Aerospace and Electronic Systems, 2001, vol. 37, no. 3, p. 914-924. DOI: $10.1109 / 7.953246$

[8] TIAN, J., CUI, W., WU, S. A novel method for parameter estimation of space moving targets. IEEE Geoscience and Remote Sensing Letters, February 2014, vol. 11, no. 2, p. 389-393. DOI: 10.1109/LGRS.2013.2263332

[9] XU, J., YU, J., PENG, Y. N., XIA, X. G. Radon-Fourier transform for radar target detection, (I): Generalized Doppler filter bank. IEEE Transactions on Aerospace Electronic Systems, 2011, vol. 47, no. 2, p. 1186-1200. DOI: 10.1109/TAES.2011.5751251

[10] XU, J., YU, J., PENG, Y. N., XIA, X. G. Radon-Fourier transform for radar target detection (III): Optimality and fast implementations. IEEE Transactions on Aerospace Electronic Systems, 2012, vol. 48, no. 2, p. 991-1004. DOI: 10.1109/TAES.2012.6178044

[11] QIONGQIONG JIA, RENBIAO WU., HAI LI. Impacts of keystone formatting on space-time adaptive processing in airborne radar. In IEEE 10th International Conference on Signal
Processing (ICSP). Bejing (China), 2010, p. 2164-2167. DOI: 10.1109/ICOSP.2010.5655762

[12] ZHOU, F., WU, R., XING, M., BAO, Z. Approach for single channel SAR ground moving target imaging and motion parameter estimation. IET Radar Sonar and Navigation, 2007, vol. 1, no. 1, p. 59-66. DOI: 10.1049/iet-rsn:20060040

[13] JUNGANG, Y., XIAOTAO, H., THOMPSON, J., TIAN, J., ZHIMIN, Z. Low-frequency ultra-wideband synthetic aperture radar ground moving target imaging. IET Radar, Sonar and Navigation, 2011, vol. 5, no. 9, p. 994-1001. DOI: 10.1049/ietrsn.2010.0387

[14] GUANGCAI SUN, MENGDAO XING, XIANG-GEN XIA, YIRONG WU, ZHENG BAO. Robust ground moving-target imaging using deramp-keystone processing. IEEE Transactions on Geoscience and Remote Sensing. Feb. 2013, vol. 51, no. 2, p. 966 to 982 . DOI: $10.1109 /$ TGRS.2012.2204889

[15] ZHOU, Z., SU, Z., WU, R. Method for detecting ground moving target with range migration. In IET International Radar Conference. 2009, vol. 141, p. 1-4.

[16] RENBIAO WU, QIONGQIONG JIA, HAI LI. A novel STAP method for the detection of fast dim air moving targets. In IEEE International Radar Conference. 2010, p. 2160-2163. DOI: 10.1109/ICOSP.2010.5655763

[17] QIONGQIONG JIA., RENBIAO WU, HAI LI. Impacts of keystone formatting on space-time adaptive processing in airborne radar. In IEEE 10th International Conference on Signal Processing (ICSP). Bejing (China), 2010, p. 2164-2167. DOI: 10.1109/ICOSP.2010.5655762

[18] QIONGQIONG JIA, RENBIAO WU. Detection of fast air moving din targets via STAP with low computation burden. In IET International Radar Conference. Xian (China), 2013, p. 1-5. DOI: 10.1049/cp.2013.0326

[19] TIAN, J., CUI, W., WU, S. A novel method for parameter estimation of space moving targets. IEEE Geoscience and Remote Sensing Letters, Feb. 2014, vol. 11, no. 2, p. 389-393. DOI: 10.1109/LGRS.2013.2263332

[20] SUN, G., XING, M.-D., WANG, Y., ZHOU, F., WU, Y., BAO, Z. Improved ambiguity estimation using a modified fractional Radon transform. IET Radar, Sonar and Navigation, 2011, vol. 5, no. 4, p. 489-495. DOI: 10.1049/iet-rsn.2010.0246

[21] WANG, Y., PENG, Y. An effective method for clutter and jamming rejection in airborne phased array radar. In IEEE International Symposium on Phased Array Systems and Technology. Boston (MA, USA), 1996, p. 349-352. DOI: 10.1109/PAST.1996.566113

[22] SHENGQI ZHU, GUISHENG LIAO, DONG YANG, HAIHONG TAO. A new method for radar high-speed maneuvering weak target detection and imaging. IEEE Geoscience and Remote Sensing Letters, July 2014, vol. 11, no. 7, p. 1175-1179. DOI: 10.1109/LGRS.2013.2283887

[23] KLEMM, R. Applications of Space-Time Adaptive Processing. The Institution of Electrical Engineers, UK, 2004. ISBN 085296 9244

[24] YANG LI, TAO ZENG, TENG LONG, ZHENG WANG. Range migration compensation and Doppler ambiguity resolution by keystone transform. In International Conference on Radar CIE 06. Shanghai, 2006, p. 1-4. DOI: 10.1109/ICR.2006.343404

[25] WANG, H., CAI, L. On adaptive spatial-temporal processing for airborne surveillance radar systems. IEEE Transactions on Aerospace and Electronic Systems, 1994, vol. 30, no. 3, p. 660-669. DOI: $10.1109 / 7.303737$

[26] HAIMOVICH, A. M., BAR NESS, Y. An eigenanalysis interference canceler. IEEE Transactions on Signal Processing, 1991, vol. 39, no. 1, p. 76-84. DOI: 10.1109/78.80767 
[27] HAIMOVICH, A. M. An eigencanceller: Adaptive radar by eigenanalysis methods. IEEE Transactions on Aerospace and Electronic Systems, 1996, vol. 32, no. 2, p. 532-542. DOI: $10.1109 / 7.489498$

[28] GOLDSTEIN, J. S., REED, I. S. Theory of partially adaptive radar. IEEE Transactions on Aerospace and Electronic Systems, 1997, vol. 33, no. 4, p. 1309-1325. DOI: 10.1109/7.625132

[29] GOLDSTEIN, J. S., REED, I. S., SCHARF, L. L. A multistage representation of the Wiener filter based on orthogonal projections. IEEE Transactions on Information Theory, 1998, vol. 44, no. 7, p. 2943-2959. DOI: $10.1109 / 18.737524$

[30] JIAN LI, GUOQING LIU, NANZHI JIANG, STOICA, P. Airborne phased array radar: clutter and jamming suppression and moving target detection and feature extraction. In Proceedings of the 2000 IEEE Sensor Array and Multichannel Signal Processing Workshop. 2000, p. 240-244. DOI: 10.1109/SAM.2000.878006

[31] ALMSLMANY, A., CAO, Q., WANG, C. A new aiborne selfprotection jammer for countering ground radars based on subnyquist. IEICE Electronic Express, 2015, vol. 12. DOI: 10.1587/elex.12.20150291

\section{About the Authors...}

Amir ALMSLMANY was borne in Alexandria Egypt, in 1982. He received the B.S. degree in Electronic Engineering and the M.Sc. degree in Communications and Electronics from the Faculty of Engineering, Alexandria University, Alexandria, Egypt, in 2003, 2011 respectively. He is currently in Ph.D. degree in the radar system in Nanjing University of Aeronautics and Astronautics, College of Electronic and Information Engineering, Nanjing, China. He also worked as an Assistant Lecturer in Air Defense College, Alexandria, Egypt. His research interests are in microwave and RF technologies and radar signal pro- cessing. He became a Member (M) of IACSIT, IEICE organization, and IEEE student member. He is a reviewer for IEEE Transaction on Signal Processing, and many other SCI journals.

Qunsheng CAO was born in China in 1959. He received his $\mathrm{Ph} . \mathrm{D}$. in Electrical Engineering from the Hong Kong Polytechnic University in 2001. From 2001 to 2005, he worked as a Research Associate in the Dept. of Electrical Engineering, University of Illinois at Urbana-Champaign and at the Army High-Performance Computing Research Center (AHPCRC), University of Minnesota. In 2005, Prof. Cao joined Nanjing University of Aeronautics and Astronautics (NUAA), China, as a Professor of Electrical Engineering. Prof. Cao's current research interests are in computational electromagnetics, and particularly in time-domain numerical techniques (FDTD, MRTD and TDFEM) for the study of microwave devices and scattering applications. Prof. Cao has published more than 120 papers in refereed journals and conference proceedings, and he is a co-author of the book Multiresolution Time Domain Scheme for Electromagnetic Engineering (Series in Microwave and Optical Engineering, vol. 1, Wiley, 2005).

Caiyun WANG was born in Shanxi, China, on September 30, 1975. She graduated in 1996 with a B.S. degree and in 1999 with a M.S. degree. She received the Ph.D. degree in Signal and Information Processing from Beihang University, Beijing, China, in 2008. She is currently an Associate Professor with the College of Astronautics, Nanjing University of Aeronautics and Astronautics (NUAA). Her major research interests are in the fields of radar automatic target recognition (RATA), radar signal processing, and adaptive signal processing, and pattern recognition. 